\title{
Role of Macrophage Migration Inhibition Factor in Kidney Disease
}

\author{
Hui Y. Lan \\ Department of Medicine and Center for Inflammatory Diseases and Molecular Therapies, The University of \\ Hong Kong, Hong Kong, SAR, China
}

\section{Key Words}

Macrophage migration inhibitory factor $\cdot$ Kidney disease $\cdot$

Macrophages $\cdot$ T cells diseases and further studies of the functional role and signaling mechanisms of MIF in human kidney diseases are needed.

Copyright $\odot 2008$ S. Karger AG, Basel

\begin{abstract}
Macrophage migration inhibitory factor (MIF) has been shown to play a pathogenic role in kidney disease. This article will review the current understanding of the expression of MIF and its functional role in immune-mediated renal injury in both human and animal models of kidney disease. Upregulation of MIF is found in both human and experimental kidney disease including renal allograft rejection and contributes significantly to macrophage and T-cell accumulation and progressive renal injury. It is now clear that MIF is a stress factor, a pro-inflammatory cytokine, a growth factor and a hormone. MIF acts through many mechanisms to mediate renal injury including the innate and adaptive immune systems, the induction of cytokines, chemokines, adhesion molecules as well as interactions with glucocorticoids and the hypothalamic-pituitary-adrenal axis. MIF exerts its biological activities via signaling through its CD74/ CD44 receptor complex to activate the downstream ERK1/2 MAP kinase. The functional importance of MIF in kidney disease is demonstrated by the findings that treatment with a neutralizing anti-MIF antibody is able to prevent or reverse renal injury in crescentic anti-GBM glomerulonephritis. In addition, mice null for MIF are protected against immunemediated lupus nephritis. MIF plays a critical role in kidney
\end{abstract}

\section{Introduction}

Macrophage migration inhibitory factor (MIF) is a pleiotropic cytokine and plays an important role in kidney disease. MIF is constitutively produced under normal circumstances but is significantly upregulated in the diseased kidney by T cells, macrophages and various nonimmune cells including tubular and glomerular epithelial cells, mesangial cells, endothelial cells, fibroblasts and vascular smooth muscle cells [1-3]. Renal MIF is released and exerts its biological activities in many pathological conditions such as septic shock, renal inflammation, immune injury and diabetes. Studies using neutralizing anti-MIF antibodies, MIF knockout mice and MIF transgenic animals demonstrate that MIF is a crucial mediator in the pathogenesis of kidney disease [4-7].

This article will review the current understanding of the general immunopathologic mechanisms of MIF in immune and inflammatory kidney diseases. Also, the signaling mechanisms whereby MIF activates T cells and macrophages, induces the expression of pro-inflammatory cytokines, and interacts with glucocorticoids will be emphasized.

\section{KARGER \\ Fax +4161306 1234 \\ E-Mail karger@karger.ch}

www.karger.com
(C) 2008 S. Karger AG, Basel

$1660-2129 / 08 / 1093-0079 \$ 24.50 / 0$

Accessible online at:

www.karger.com/nee
Prof. Hui Y. Lan, MD, PhD

Department of Medicine, The University of Hong Kong

8/F-39, Research Block, 21 Sassoon Road

Pokfulam, Hong Kong, SAR (China)

E-Mail hylan@hku.hk 


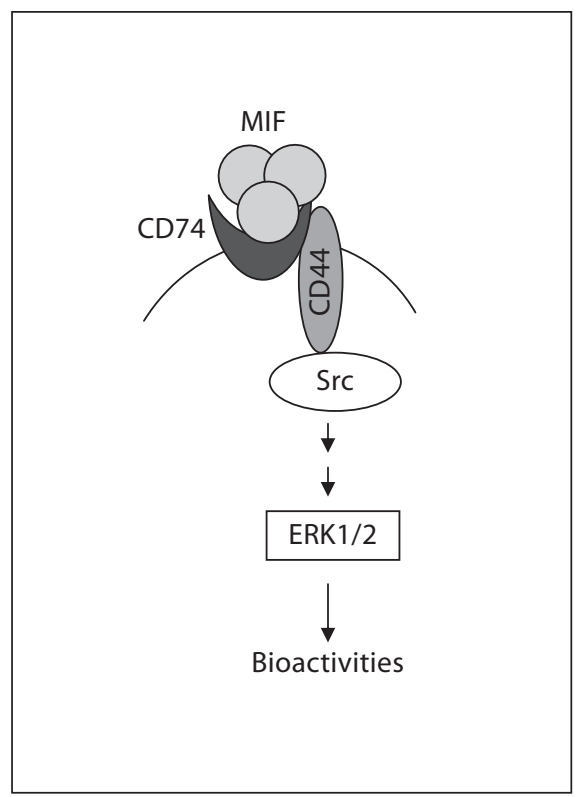

Fig. 1. MIF signaling pathways. MIF binds to CD74 that recruits CD44 to form the receptor complex with resultant activation of Src-family kinase and the downstream ERK1/2 MAP kinase signaling pathway.

\section{MIF, MIF Receptor and the Signaling Pathway}

Major progress in understanding the functional role of MIF has been made on the basis of the identification of the MIF receptor CD74 [8], which is the cell-surface form of the MHC class II invariant chain. Although CD74 functions as a cell-surface binding site for MIF, it does not have a typical signal-transducing cytosolic domain and further work indicates that the signal transduction of MIF requires the recruitment and activation of CD44, a genetically polymorphic molecule with an important role in cell-extracellular matrix interaction [9]. As shown in figure 1, it is now clear that CD44 acts as an integral member of the CD74 receptor complex leading to MIF signal transduction. Furthermore, MIF-induced ERK1/2 MAP kinase phosphorylation through CD44 also requires the Src tyrosine kinase [9]. Several of the biologic activities of MIF have been shown to proceed via activation of ERK1/2 MAP kinase. These include modulation of arachidonic acid metabolism and prostaglandin production, regulation of p53 activity and the intracytoplasmic action of c-Jun activation domain binding protein-1, and the activation of additional ERK effectors, such as the Ets family of transcription factors that regulate toll-like receptor expression [reviewed in 10]. Like MIF, upregula- tion of both MHC class II and CD44 is found in the diseased kidney including glomerular cells, tubulointerstitial cells and infiltrating $\mathrm{T}$ cells and macrophages that contribute significantly to the severity of renal inflammation and functional impairment [11]. Thus, coexpression of MIF and its receptor complex CD74 and CD44 in the diseased kidney suggests that MIF produced by inflammatory $\mathrm{T}$ cells and macrophages as well as intrinsic kidney cells during renal inflammation may mediate progressive renal injury via the CD74/CD44 receptor complex in a paracrine and autocrine manner.

\section{MIF Is Upregulated in Both Human and Experimental Kidney Disease}

MIF mRNA and protein is constitutively expressed in many cells in the normal kidney, including glomerular and tubular epithelial cells, endothelial cells, fibroblasts and vascular smooth muscle cells $[1,2]$. MIF is markedly upregulated in disease conditions such as experimental and human glomerulonephritis and renal allograft rejection [1-3]. Indeed, strong MIF expression by glomerular and tubulointerstitial cells is tightly associated with profound macrophage and T-cell accumulation and contributes to focal glomerular and tubulointerstitial damage and particularly glomerular crescent formation. Correlation analysis shows that the level of MIF in both human and experimental kidney diseases including renal allograft rejection is correlated closely with the number of infiltrating macrophages and $\mathrm{T}$ cells, resulting in progressive renal dysfunction such as proteinuria, an increased serum creatinine and a fall of glomerular filtration rate. The pathogenic role of upregulation of MIF in the development of kidney injury is evident in MIF transgenic mice in which conditional expression of MIF in podocytes leads to the development of severe proteinuria and glomerulosclerosis, resulting in end-stage kidney disease [6].

\section{MIF in T-Cell-Mediated Kidney Disease}

MIF is produced by activated T cells. In turn, MIF also causes $\mathrm{T}$ cell activation via a paracrine and autocrine manner in kidney diseases such as glomerulonephritis and renal allograft rejection. Evidence for a key role of MIF in T-cell-mediated kidney disease includes the observation that MIF-producing $\mathrm{T}$ cells are exclusively localized to the area of severe tissue injury, including glo- 
merular crescentic formation $[1,2]$, focal glomerular and tubulointerstitial lesions [2], and necrotic vascular inflammation [3]. Furthermore, T-cell-mediated renal injury is prevented in lupus-prone mice targeted for the deletion of MIF whilst administration of anti-MIF antibody is protective in a rat model of anti-GBM crescentic glomerulonephritis, thereby demonstrating a critical role for MIF in T-cell-mediated kidney disease [4, 7].

There are several possible mechanisms in the regulation of T-cell-mediated immune injury by MIF. First, MIF may have a direct or indirect role in recruiting $\mathrm{T}$ cells to sites of immune and inflammatory injury. For example, upregulation of chemokines and adhesion molecules is induced by MIF. Second, MIF may act by stimulating T-cell proliferation and activation to mediate renal injury via the delayed-type hypersensitivity (DTH) mechanism. MIF is important in the T-cell-mediated DTH response that is a key mechanism in the pathogenesis of many immune diseases including immunologically-mediated glomerulonephritis and allograft kidney rejection. Indeed, MIF is the first T-cell cytokine identified in association with DTH response. Direct evidence for a role of MIF in the DTH response associated with kidney disease comes from the findings that neutralizing anti-MIF antibody treatment of rats with primed rabbit anti-GBM glomerulonephritis inhibits an antigen-challenge skin DTH reaction induced by the intradermal injection of rabbit IgG [4].

\section{MIF in Macrophage-Mediated Kidney Diseases}

Macrophages are the major cell type that produces MIF and are found to contain significant amounts of preformed MIF protein within intracellular pools. We find that macrophages produce MIF in both experimental and human kidney disease including renal allograft rejection [1-5]. Indeed, upregulation of MIF is tightly associated with macrophage accumulation and the severity of tissue injury including glomerular crescent formation, suggesting that local production of MIF by macrophages may, in turn, activate macrophages to produce cytokines (IL-1, TNF- $\alpha$, IL-2, INF- $\gamma$ ), chemokines (MCP-1) and adhesion molecules (ICAM-1). Evidence to support a critical role for MIF in macrophage-mediated renal injury also comes from the finding that administration of a neutralizing anti-MIF antibody is able to prevent or reverse macrophage accumulation and activation in a rat model of crescentic glomerulonephritis $[4,5]$.

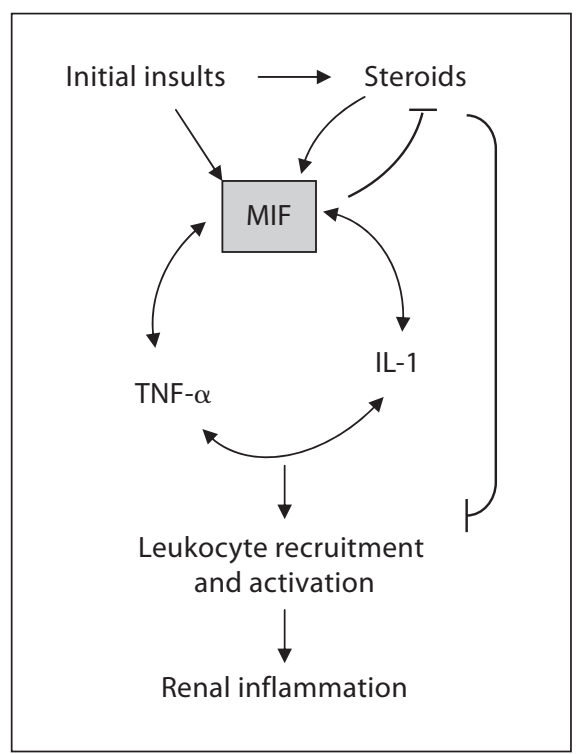

Fig. 2. Role of MIF in the inflammatory cascade. MIF, an upstream mediator of inflammatory cascade, is rapidly released from intracellular pools in response to renal injury and steroids. Once released, MIF initiates the inflammatory cascade by inducing pro-inflammatory cytokines including IL-1 and TNF- $\alpha$ which in turn further stimulate MIF production. MIF also antagonizes the anti-inflammatory or immunosuppressive effects of glucocorticoids to promote ongoing renal inflammation and immune responses.

\section{MIF Acts as a Critical Pro-Inflammatory Cytokine and a Glucocorticoid Antagonist in Kidney Disease}

MIF is an established pro-inflammatory cytokine in kidney disease and, unlike classical inducible pro-inflammatory cytokines such as IL- 1 and TNF- $\alpha$, MIF is preformed and stored within the cytoplasm of macrophages and other intrinsic cells within the kidney (fig. 2). MIF is released upon stimulation by cellular stress, endotoxin, exotoxin, infection, inflammation and immune responses. Once released, MIF acts as a pro-inflammatory cytokine to induce expression of other inflammatory cytokines/mediators including IL-1, TNF- $\alpha$, IL-2, IL-6, IL8 , INF- $\gamma$, and iNOS, with these mediators further promoting inflammatory and immune responses. Thus, MIF is a critical mediator and sits upstream in the inflammatory cascade as demonstrated by studies using neutralizing anti-MIF antibody. Treatment of immunologically-induced anti-GBM glomerulonephritis with an anti-MIF antibody prevents and reverses disease in association with a marked reduction of pro-inflammatory cytokines including IL-1 and TNF- $\alpha[4,5]$, reinforcing the 
key, upstream role of MIF in pro-inflammatory cytokine expression during the pathogenesis of glomerulonephritis. Furthermore, TNF- $\alpha$ blockade by administration of soluble TNF- $\alpha$ receptors in anti-GBM glomerulonephritis inhibits renal MIF expression and progressive renal injury, demonstrating a pathogenic role of MIF in the inflammatory cascade [12]. Thus, MIF acts not only as a key upstream cytokine in the pro-inflammatory cytokine cascade with MIF-mediated induction of IL-1 and TNF$\alpha$ production, but also acts as a pro-inflammatory cytokine in response to other pro-inflammatory stimuli.

Unlike other pro-inflammatory cytokines that are generally suppressed by the anti-inflammatory actions of glucocorticoids, MIF is induced by glucocorticoids and acts as an antagonist of glucocorticoid actions within the immune system (fig. 2). Glucocorticoid-induced secretion of MIF is tightly regulated and follows a bell-shaped dose-response curve. Low, physiologic amounts of glucocorticoids stimulate MIF release from murine monocytes and macrophages, with MIF release inhibited at high, anti-inflammatory concentrations of glucocorticoids $\left(>10^{-8} \mathrm{M}\right)$ [13]. Thus, MIF-induced renal injury may be associated with an antagonistic action upon the anti-inflammatory and immunosuppressive effects of glucocorticoids. For example, the reversal of established antiGBM crescentic glomerulonephritis after treatment with a MIF neutralizing antibody is associated with an increase in serum levels of glucocorticoids, and this may be the result of the counter-regulating interaction between MIF and glucocorticoids in kidney disease.

Although the mechanisms by which MIF antagonizes glucocorticoid actions remain largely unclear, the relationships between hypothalamic-pituitary-adrenal (HPA) hormones and immunity have received special attention due to the importance of these hormones in the stress response and autoimmune diseases. MIF is rapidly released following activation of the HPA system along with glucocorticoids in response to stimuli. MIF may antagonize the action of glucocorticoids by several mechanisms [reviewed in 14]. First, MIF upregulates cytoplasmic phospholipase $A_{2}$, which is an important target for the anti-inflammatory actions of glucocorticoids. Second, MIF may antagonize the anti-inflammatory inhibition of NF- $\kappa \mathrm{B}$ activation by glucocorticoids. Glucocorticoids prevent the activation of NF- $\kappa \mathrm{B}$, in part, by increasing the expression of ІкB which maintains NF- $\kappa \mathrm{B}$ in an inactive form in the cytosol. MIF may antagonize this effect by overriding the glucocorticoid-mediated increase in the cytosolic concentration of IкB. Thus, by blocking glucocorticoid-induced IкB synthesis, MIF promotes the translocation of NF- $\mathrm{KB}$ into the nucleus, thereby activating key pro-inflammatory pathway involved in mediating renal injury. Finally, MIF is capable of antagonizing glucocorticoid induction of MAP kinase phosphatase, which is important in downregulating the MAP-kinaseassociated inflammatory response. Taken together, the antagonistic effect on the anti-inflammatory activities of glucocorticoids may be a key mechanism by which MIF mediates renal injury.

\section{Prospective Studies of MIF in Kidney Diseases}

Increasing evidence from both experimental and human kidney disease has shown that MIF is a critical mediator of renal injury. It is clear that MIF acts through many mechanisms to mediate kidney disease including innate and adaptive immunity, induction of cytokines, chemokines, adhesion molecules, and interactions with glucocorticoids and the HAP axis. Despite this, future studies are needed to dissect the signaling pathways of MIF in kidney diseases. The recent discovery of the MIF receptor complex (CD74/CD44) has provided an important tool for such studies. Furthermore, although we have demonstrated the efficacy of anti-MIF treatment in kidney disease using a monoclonal neutralizing antibody, it is necessary to develop new therapeutic strategies to specifically block MIF with small molecules or chemical MIF inhibitors. In addition, it is also important to investigate the counter-regulatory mechanisms of MIF in patients with steroid resistance because MIF can antagonize the beneficial actions of steroids. Indeed, there is a MIF allele polymorphism associated with steroid resistance in patients with nephrotic syndrome [15]. Thus, inhibition of MIF may offer an attractive therapeutic strategy because it would act uniquely to inhibit the antagonistic effects of MIF upon endogenous or prescribed glucocorticoids and thereby provide a new approach for the treatment of kidney diseases in patients who do not respond to steroid therapy.

\section{Acknowledgement}

This work was supported by a grant from the Research Grants Committee of Hong Kong (RGC CERG 759206). 


\section{References}

- 1 Lan HY, Mu W, Yang N, Meinhardt A, Nikolic-Paterson DJ, Ng YY, Bacher M, Atkins RC, Bucala R: De novo renal expression of macrophage migration inhibitory factor during the development of rat crescentic glomerulonephritis. Am J Pathol 1996;149: 1119-1127.

-2 Lan HY, Yang N, Nikolic-Paterson DJ, Yu XQ, Mu W, Isbel NM, Metz CN, Bucala R, Atkins RC: Expression of macrophage migration inhibitory factor in human glomerulonephritis. Kidney Int 2000;57:499-509.

-3 Lan HY, Yang N, Brown FG, Isbel NM, Nikolic-Paterson DJ, Mu W, Metz CN, Bacher M, Atkins RC, Bucala R: Macrophage migration inhibitory factor expression in human renal allograft rejection. Transplantation 1998;66:1465-1471.

4 Lan HY, Bacher M, Yang N, Mu W, NikolicPaterson DJ, Metz C, Meinhardt A, Bucala R, Atkins RC: The pathogenic role of macrophage migration inhibitory factor in immunologically induced kidney disease in the rat. J Exp Med 1997;185:1455-1465.

5 Yang N, Nikolic-Paterson DJ, Ng YY, Mu W, Metz C, Bacher M, Meinhardt A, Bucala R, Atkins RC, Lan HY: Reversal of established rat crescentic glomerulonephritis by blockade of macrophage migration inhibitory factor (MIF) - potential role of MIF in regulating glucocorticoid production. Mol Med 1998;4:413-424.
-6 Sasaki S, Nishihira J, Ishibashi T, Yamasaki Y, Obikane K, Echigoya M, Sado Y, Ninomiya Y, Kobayashi K: Transgene of MIF induces podocyte injury and progressive mesangial sclerosis in the mouse kidney. Kidney Int 2004;65:469-481.

7 Hoi AY, Hickey MJ, Hall P, Yamana J, O’Sullivan KM, Santos LL, James WG, Kitching AR, Morand EF: Macrophage migration inhibitory factor deficiency attenuates macrophage recruitment, glomerulonephritis, and lethality in MRL/lpr mice. J Immunol 2006;177:5687-5696.

$>8$ Leng L, Metz CN, Fang Y, Xu J, Donnelly S, Baugh J, Delohery T, Chen Y, Mitchell RA, Bucala R: MIF signal transduction initiated by binding to CD74. J Exp Med 2003;197: 1467-1476.

9 Shi X, Leng L, Wang T, Wang W, Du X, Li J, McDonald C, Chen Z, Murphy JW, Lolis E, Noble P, Knudson W, Bucala R: CD44 is the signaling component of the macrophage migration inhibitory factor-CD74 receptor complex. Immunity 2006;25:595-606.
10 Bucala R, Donnelly SC: Macrophage migration inhibitory factor: a probable link between inflammation and cancer. Immunity 2007;26:281-285.

11 Kirimca F, Sarioğlu S, Camsari T, Kavukçu S: Expression of CD44 and major histocompatibility complex class II antigens correlate with renal scarring in primary and systemic renal diseases. Scand J Urol Nephrol 2001;35: 509-514.

$>12$ Lan HY, Yang N, Metz C, Mu W, Song Q, Nikolic-Paterson DJ, Bacher M, Bucala R, Atkins RC: TNF- $\alpha$ up-regulates renal MIF expression in rat crescentic glomerulonephritis. Mol Med 1997;3:136-144.

13 Calandra T, Bernhagen J, Metz CN, Spiegel LA, Bacher M, Donnelly T, Cerami A, Bucala R: MIF as a glucocorticoid-induced modulator of cytokine production. Nature 1995;377:68-71.

14 Flaster H, Bernhagen J, Calandra T, Bucala $\mathrm{R}$ : The macrophage migration inhibitory factor-glucocorticoid dyad: regulation of inflammation and immunity. Mol Endocrinol 2007;21:1267-1280.

-15 Berdeli A, Mir S, Ozkayin N, Serdaroglu E, Tabel Y, Cura A: Association of macrophage migration inhibitory factor $-173 \mathrm{C}$ allele polymorphism with steroid resistance in children with nephrotic syndrome. Pediatr Nephrol 2005;20:1566-1571. 